https://creativecommons.org/licenses/by/4.0/

\title{
OPUSCULO SOBRE LA SOLUCIÓN DE LA ECUACIÓN CÚBICA
}

\author{
Opuscle on the solution of the equation of third degree
}

CARLOS M. MATA RODRÍGUEZ ${ }^{1}$

$\underline{\text { Recibido:5 de diciembre de 2020. Aceptado:13 de enero de } 2021}$

DOI: http://dx.doi.org/10.21017/rimci.2021.v8.n15.a93

\begin{abstract}
Resumen
En la teoría de las ecuaciones, la solución de la ecuación de tercer grado (cúbica) ocupa un lugar destacado en la historia de las matemáticas. Notables matemáticos realizaron importantes aportes para lograr completar su teoría, la cual no estuvo exenta de graves conflictos personales referentes a la adjudicación de las fórmulas que permitían determinar su solución. El presente artículo muestra un detallado análisis en referencia a la ecuación de tercer grado, tanto histórico como analítico.
\end{abstract}

Palabras clave. Ecuación de tercer grado; discriminante; números complejos; raíces.

\section{Abstract}

In the theory of equations, the solution of the equation of third degree (cubic) occupies a prominent place in the history of mathematics. Notable mathematicians made important contributions to complete that theory, which was not exempt from serious personal conflicts regarding the adjudication of the formulas that allowed to determine its solution. This article shows a detailed analysis in reference to the third-degree equation, both historical and analytical.

Key words. Cubic equation; discriminating; roots; complex number.

\section{INTRODUCCIÓN}

L OS PRIMEROS intentos por resolver las ecuaciones de tercer grado (cúbicas) aparecen en los trabajos del matemático italiano Scipio Ferro (1425-1526) [1] cuando ejercía como profesor de matemáticas en la ciudad italiana de Bologna, proponiendo la solución general de la ecuación $a x^{3}+b x^{2}+c x+d=0$ a la forma transformada $x^{3}$ $+p x+q=0(a, b>0)$ con la eliminación del término cuadrático, hacia el año 1500, dando a conocer el resultado de sus investigaciones en los años finales de su vida.

Por otra parte el destacado matemático italiano Nicolo de Brescia (1505-1557) conocido como Tartaglia, [2] logra resolver otras variantes de dicha ecuación.

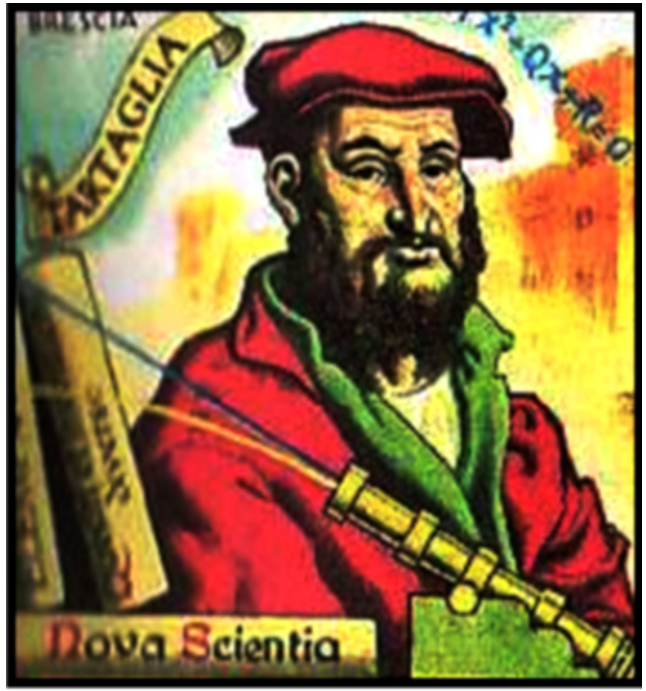

Tartaglia

1 Profesor. Licenciado en Matemáticas, Universidad Máximo Gómez Báez. Ciego de Ávila, Cuba. Correo electrónico: cmatas1010@gmail.com. ORCID: https://orcid.org/0000-0003-0734-2612 
El también matemático italiano Hieronimo Cardano (1501-1576) le solicita a Tartaglia la fórmula de solución con el marcado compromiso de que no fuese revelada. No obstante Cardano la publica en su célebre libro Ars Magna en el año 1545[3][4].

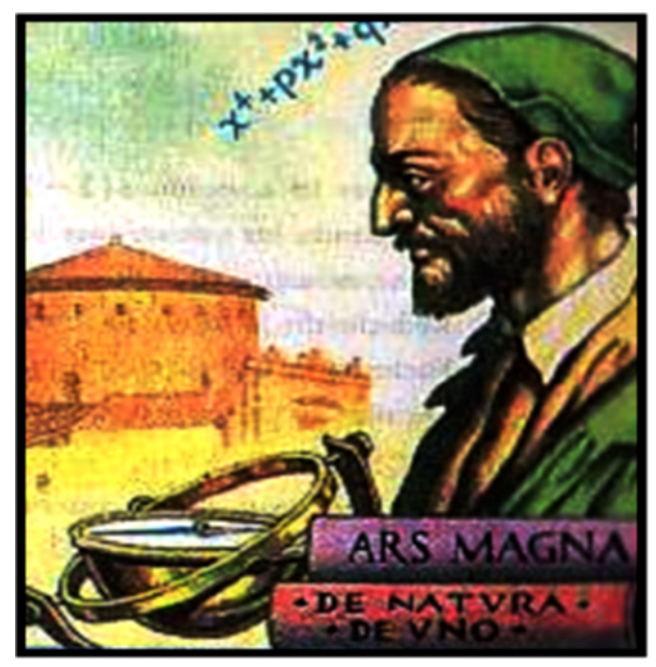

Cardano

HIERONYMI CAR DANI, PRASTANTISSIMI MATHE

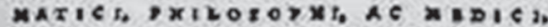
ARTIS MAGNE, SIVB DE RBGVLIS ALGEBRAICI8, Libunus. Qel\& cochus operia de Avidhoeta, guod OPVS PBRFBCTVM

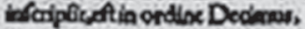

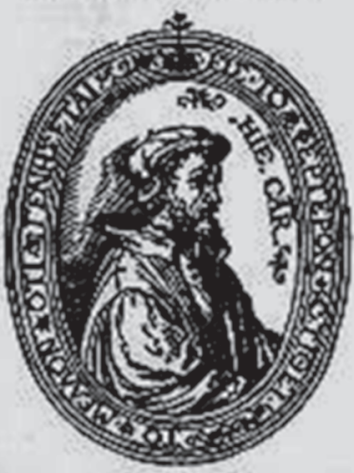

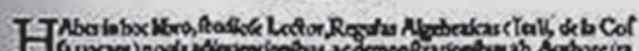

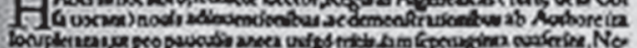

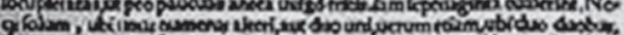

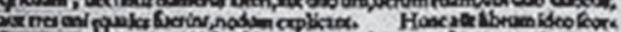

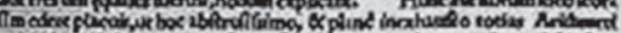

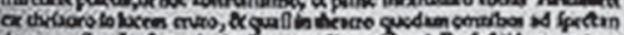

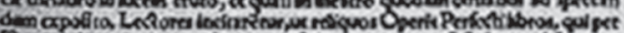

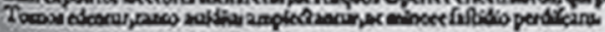

De ahí el nombre de fórmula de Cardano. La fórmula en cuestión es:

$$
\begin{gathered}
x=\sqrt[3]{\left(\left(\frac{-q}{2}+\sqrt{\Delta}\right)\right)}+\sqrt[3]{\left(\left(\frac{-q}{2}-\sqrt{\Delta}\right)\right)} \\
\text { Siendo } \Delta \text { el determinante } \Delta=\frac{q^{2}}{4}+\frac{p^{3}}{27}
\end{gathered}
$$

Las disputas en torno a la primacía de haber resuelto la ecuación de tercer grado generaron grandes controversias y debates públicos en aquella época.

En Ars Magna aparece por primera vez el concepto de raíz múltiple. El primer ejemplo que proporciona Cardano de una ecuación polinomial con este tipo de raíz es $\times 3=12 x+16$, siendo ?2 es una raíz doble.

A pesar del gran aporte matemático que constituía la fórmula, Cardano y sus discípulos no tardaron en darse cuenta que para un determinado tipo de ecuación, la fórmula obtenida no podía ser aplicada. Cardano se vio impulsado al estudio de trece tipos diferentes de ecuaciones cúbicas.

Este es el denominado caso irreducible de la fórmula de Cardano. (raíces cuadradas de números negativos). El matemático italiano Raphael Bombelli (1526-1573) estudia la ecuación y publica en 1572 un tratado de Algebra donde se hace mención por primera vez de la teoría de los números complejos mostrando empíricas soluciones para la ecuación cúbica.

La solución de la anterior problemática la tiene el matemático francés Francois Vieta (1540-1603).

Dándole solución al caso irreducible evitando las expresiones imaginarias mediante métodos trigonométricos [5].

En la búsqueda de la solución de ecuaciones de mayor grado transcurrieron más de 300 años. Siendo notable los trabajos realizados por Paolo Ruffini (1765-1822) en este tema. Sólo en el siglo XIX el gran matemático noruego Niels Henrik Abel, (1802-1829) demostró en 1823 que las 


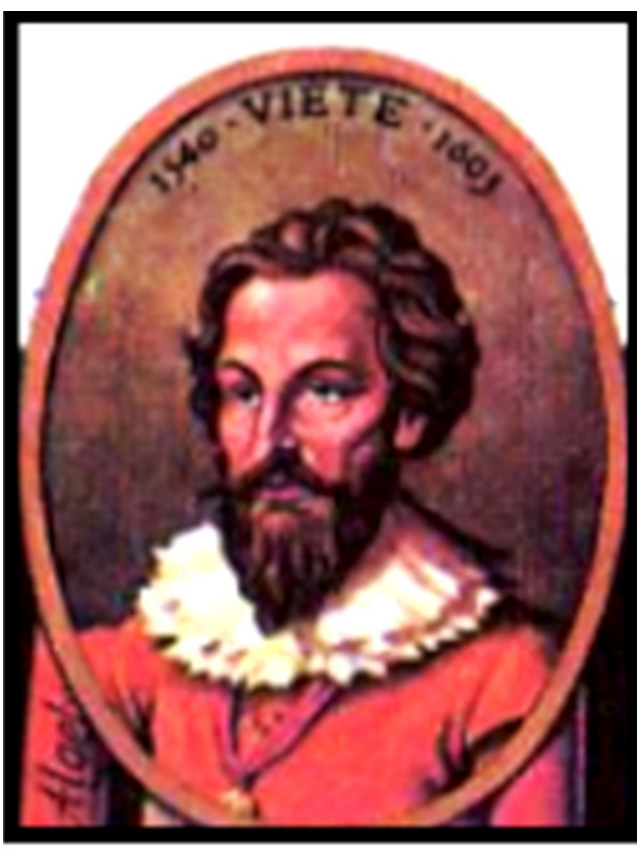

Vieta

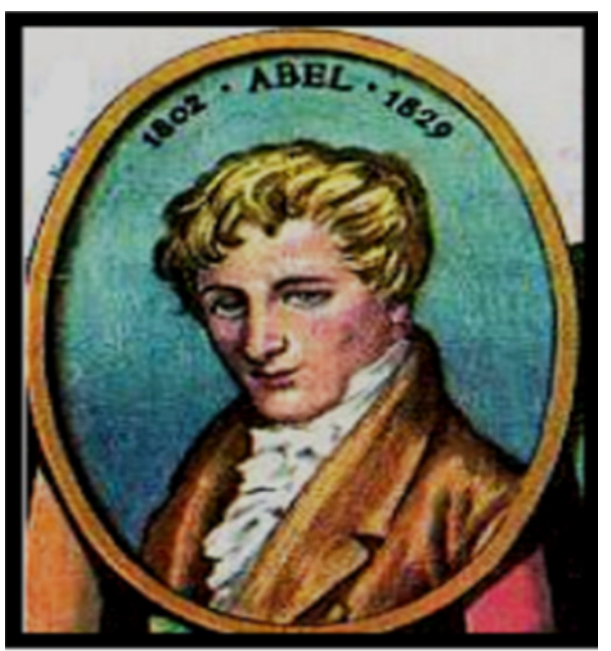

Abel

ecuaciones de grado $n>4$ no podían ser resueltas por medio de radicales.

\section{Desarkollo}

Vamos a exponer dos ejemplos demostrativos para explicar cómo se determina la solución de una ecuación de tercer grado, aplicando la fórmula de Tartaglia-Cardano y el procedimiento trigonométrico creado por Vieta.
La ecuación general de tercer grado, tiene una estructura algebraica de la forma:

$$
\begin{aligned}
& a x^{3}+b x^{2}+c x+d=0 \\
& \text { donde } a, b, c, d \varepsilon R, a \neq 0
\end{aligned}
$$

Para proceder al cálculo de su solución, comencemos por transformar la ecuación (3) en otra, en la cual el coeficiente de $x^{3}$ sea la unidad y que el término en $x^{2}$ no exista. Realizada esta operación, establecemos en (3) la sustitución.

$$
x=z-\frac{b}{3 a}
$$

Que al efectuar las correspondientes simplificaciones, llegamos a la ecuación cúbica de la forma:

$$
x^{3}+p x+q=0
$$

la cual se resolverá en un determinado caso por la fórmula (1) en dependencia de un valor numérico denominado discriminante de la ecuación [6][7].

En este punto de la teoría, consideramos oportuno la solución del ejemplo demostrativo número 1.

Sea la ecuación:

$$
x^{3}+2 x^{2}+3 x+4=0
$$

Aplicando (4) tenemos que $b / 3 a=2 / 3$

Haciendo $t=2 / 3 y$

Sustituyendo en la ecuación, siendo

$$
\begin{aligned}
& a=1 \quad b=2 \quad c=3 \quad d=4 \\
& a\left(z-\frac{b}{3 a}\right)^{3}+b\left(z-\frac{b}{3 a}\right)^{2}+c\left(z-\frac{b}{3 a}\right)+d=0
\end{aligned}
$$

Una vez simplificada la expresión algebraica, llegamos a:

$$
z^{3}+\frac{5 z}{3}+\frac{70}{27}
$$


Teniendo en cuenta la forma canoníca (5) $\mathrm{p}$ $=5 / 3$ y q $=70 / 27$

Con los valores obtenidos, calculamos el determinante de la ecuación, aplicando

$$
\Delta=\frac{q^{2}}{4}+\frac{p^{3}}{27}=1.852
$$

Siendo $\Delta>0$

A continuación, aplicamos (1) y obtenemos el primer valor $\mathrm{z}$

$$
z=\sqrt[3]{\left(\left(\frac{-q}{2}+\sqrt{\Delta}\right)\right)}+\sqrt[3]{\left(\left(\frac{-q}{2}-\sqrt{\Delta}\right)\right)}=-0.984
$$

La primera solución real de la ecuación es:

$x_{1}=z-t=-1.651$

Ahora tenemos que hallar las dos restantes soluciones, esto es, $\mathrm{x}_{2} \mathrm{y}_{3}$.

Pues debemos tener en cuenta que:

Si $\Delta>0$ la ecuación tiene una raíz real y dos imaginarias conjugadas.

Por lo que $\mathrm{x}_{2} \mathrm{y} \mathrm{x}_{3}$ son del tipo [8]

\section{$\alpha \pm \beta \mathrm{i}$}

Raíces imaginarias conjugadas, para determinarlas aplicamos.

$$
\begin{aligned}
& \delta 1=\sqrt[3]{\left(\left(\frac{-q}{2}+\sqrt{\Delta}\right)\right)}=0.401 \\
& \delta 2=\sqrt[3]{\left(\left(\frac{-q}{2}-\sqrt{\Delta}\right)\right)}=-1.385 \\
& \alpha=\frac{-\delta 1}{2}-\frac{\delta 2}{2}-t=-0.175 \\
& \beta=\frac{\sqrt{3}}{2}(\delta 1-\delta 2) \sqrt{-1}=1.547 i
\end{aligned}
$$

Siendo la solución de la ecuación

$$
\begin{aligned}
& x^{3}+2 x^{2}+3 x+4=0 \\
& x_{1}=-1.651 \\
& x_{2}=-0.175+1.547 i \\
& x_{3}=-0.175-1.547 i
\end{aligned}
$$

La representación gráfica de la ecuación se muestra en la figura 1.

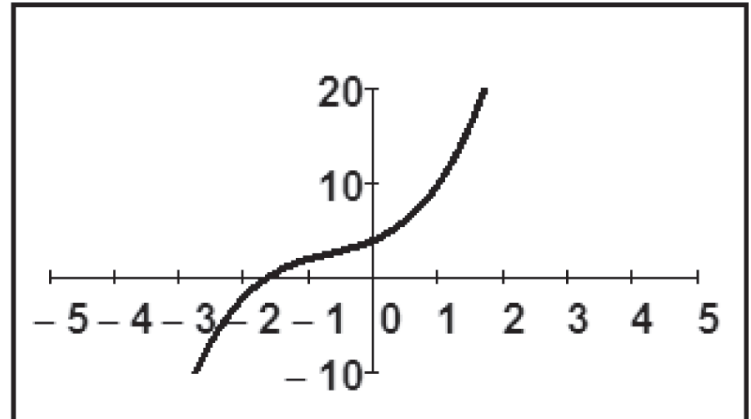

Figura 1.

Ejemplo demostrativo número 2.

Ecuación,

$$
2 x^{3}-13 x^{2}-59 x+280=0
$$

$$
\begin{aligned}
& \mathrm{t}=\frac{-13}{6} \\
& 2(\mathrm{z}-\mathrm{t})^{3}-13(\mathrm{z}-\mathrm{t})^{2}-59(\mathrm{z}-\mathrm{t})+280=0 \\
& \Delta=\frac{\mathrm{q}^{2}}{4}+\frac{\mathrm{p}^{3}}{27}=-2289.422 \\
& \mathrm{z}^{3}-\frac{523 \mathrm{z}}{12}+\frac{3010}{54}=0 \\
& \mathrm{p}=\frac{-523}{12} \\
& \mathrm{q}=\frac{3010}{54}
\end{aligned}
$$

Siendo $\Delta<0$ existen tres raíces reales distintas $y$ es conocido con el nombre de caso 
irreducible, porque antes de la invención de los números complejos no se podía resolver [9].

Aplicando el método trigonométrico de Vieta

$$
\begin{aligned}
\rho & =\sqrt{\frac{-\mathrm{p}^{3}}{27}}=55.373 \\
\theta & =\operatorname{acos}\left(\frac{-\mathrm{q}}{2 \rho}\right)=2.098 \\
\mathrm{x} 1 & =2 \sqrt[3]{\rho} \cos \left(\frac{\theta}{3}\right)=5.833 \\
\mathrm{x} 2 & =2 \sqrt[3]{\rho} \cos \left(\frac{\theta}{3}+120 \frac{\pi}{180}\right)=-7.167 \\
\mathrm{x} 3 & =2 \sqrt[3]{\rho} \cos \left(\frac{\theta}{3}+240 \frac{\pi}{180}\right)=1.333
\end{aligned}
$$

Obtenemos las soluciones reales.

$$
\begin{aligned}
& \mathrm{R} 1=\mathrm{x} 1-\mathrm{t}=8 \\
& \mathrm{R} 2=\mathrm{x} 2-\mathrm{t}=-5 \\
& \mathrm{R} 3=\mathrm{x} 3-\mathrm{t}=3.5
\end{aligned}
$$

La representación gráfica de la ecuación se muestra en la figura 2.

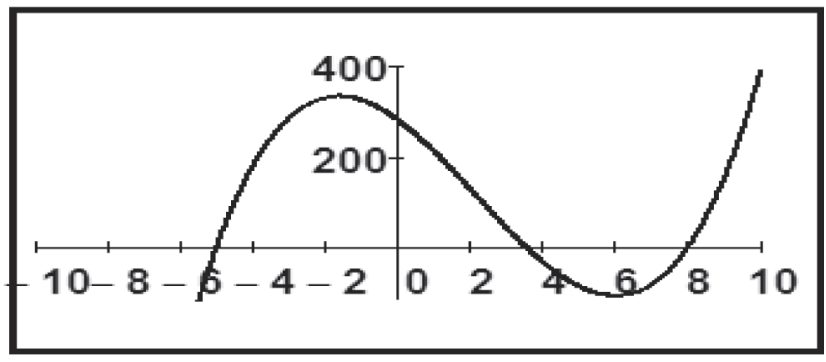

Figura 2.

Por último, si $\Delta=0$ podemos utilizar las fórmulas del ejemplo número 2, pues estamos en presencia de tres soluciones reales de las cuales dos son iguales.

\section{CONCLUSIONES}

La solución de la ecuación de tercer grado, como hemos podido ver en el desarrollo de este opúsculo ha sido durante siglos motivo de estudio de destacados matemáticos [10]. Tartaglia y Cardano para resolver este tipo de ecuaciones, formularon esencialmente fórmulas análogas a la de la ecuación de segundo grado, si bien mucho más complicadas. Si el lector desea continuar con el estudio de la Teoría de las Ecuaciones, le recomendamos la lectura de los artículos "Solución trigonométrica de la ecuación de segundo grado" y "El método de Newton-Raphson para la obtención de raíces mediante programación MATHCAD" en la Revista Ingeniería, Matemáticas y Ciencias de la Información, volumen 4, número 8. JulioDiciembre de 2017, y volumen 4, número 7. Enero-Junio de 2017 [11] [12].

Con el empleo de software matemático, la solución de la ecuación cúbica, ha dejado de ser una tarea compleja, por ejemplo, la ecuación que sirvió de base para el primer ejemplo del presente artículo resuelta con MATHCAD, [13] tiene por soluciones, las mostradas en la figura número 3.

\section{REFERENCIAS}

[1] Herbert Hawkes, Higher Algebra, Ginn and Company, New York, 1913.

[2] Raymond Brink, Algebra College Course, Appleton Century Company, New York, 1934.

[3] K, Ribnikov, Historia de las Matemáticas, Moscú, MIR, 1980.

[4] Florian Cajori, A history of Mathematics,, The Macmillan Company, 1909. New York

[5] J.H. Hotmann, Historia de las Matemáticas, México, LIMUSA, 2002.

[6] H.S.Hall and S.R. Knigth, Algebra Superior, México, UTEHA, 1948.

[7] Julio Rey Pastor, Elementos de Análisis Algebraico, Buenos Aires, Ed. Kapelusz, 1939.

[8] Pablo Miquel Merino, Elementos de Algebra Superior, La Habana, CULTURAL S.A. 1940.

[9] Gerhard Hessenberg, Trigonometría Plana y Esférica, Barcelona, Ed. Labor, 1929.

[10] Richart Courant, Herbert Robbins, Qué es la matemática?, Madrid, AGUILAR, 1955. 
[11] C. Mata Rodriguez, Solución como función lineal aproximada en un reducido intervalo de ecuaciones diferenciales de primer orden con fundamento en el problema de cauchy, RIMCI, vol. 5, n. ${ }^{\circ} 10$, pp. 33-37, jul. 2018.
[12] C. M. Mata rodríguez, «El método de NewtonRaphson para la obtención de raíces de ecuaciones mediante programación en MATHCAD. Algoritmo de cálculo», RIMCI, vol. 4, n. ${ }^{\circ}$ 7, ene. 2017.

[13] MATHCAD, version 14.0.0.163. 2007 Parametric Technology Corporation. 


\section{ANEXO}

Solución de la ecuación cúbica, mediante programación en MATHCAD
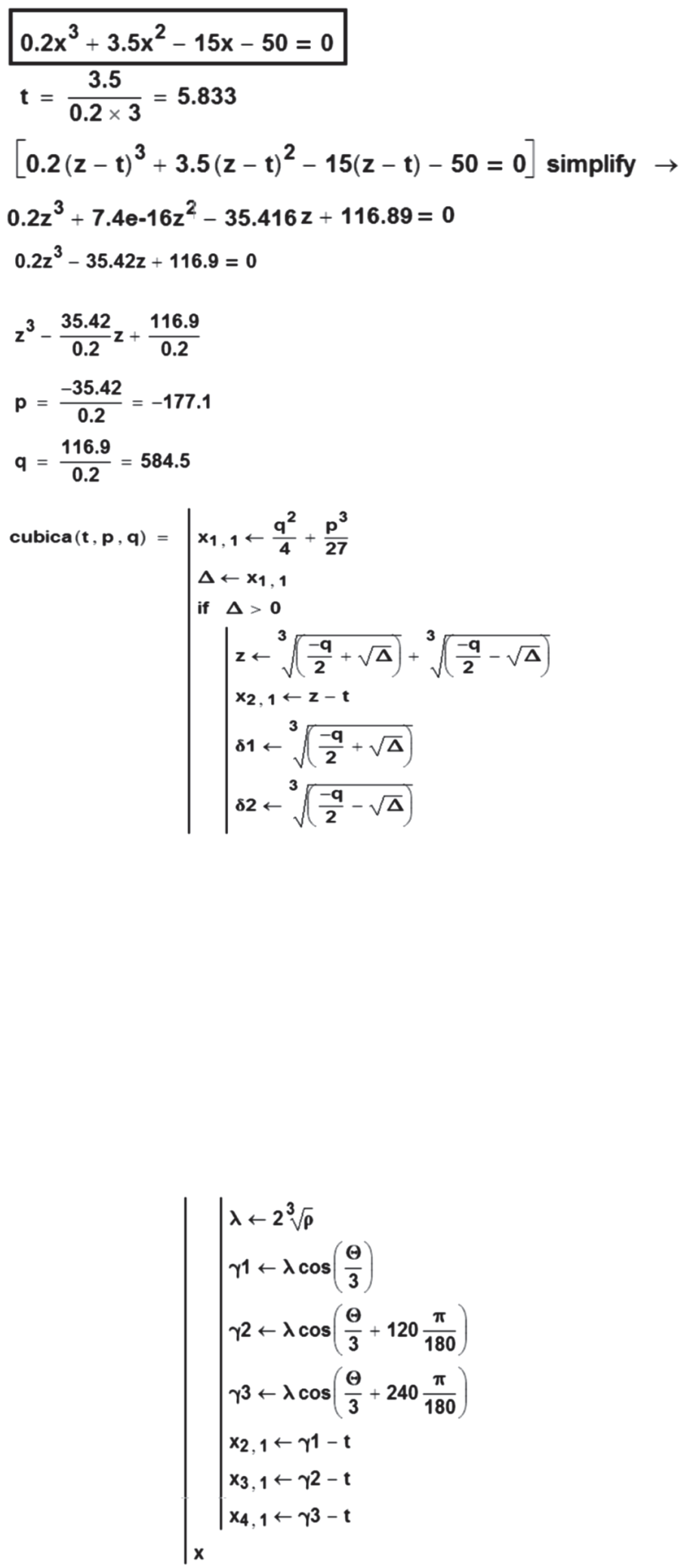
